\title{
First Principles Determination of Electronic Excitations Induced by Charged Particles
}

\author{
David B. Lingerfelt, ${ }^{*}$ Panchapakesan Ganesh, ${ }^{*}$ Jacek Jakowski,, and Bobby \\ Sumpter
}

\author{
Nanomaterials Theory Institute, \\ Center for Nanophase Materials Sciences, \\ Oak Ridge National Laboratory, \\ Oak Ridge, TN
}

E-mail: LingerfeltDB@ornl.gov; GaneshP@ornl.gov; JakowskiJ@ornl.gov

\begin{abstract}
Time-dependent density functional theory (TD-DFT) is nowadays routinely applied to molecular and nanoscaled condensed-phase materials for the calculation of electronic excitation energies and their associated optical transition probabilities. In this paper, we derive and implement expressions within the linear response TD-DFT framework for rates of transition between the ground and excited states induced by an external point charge. Symmetry considerations are given for the coupling between electronic states of well defined parity in two extreme limits of the point charge's position, and a general method to determine the range of point charge positions over which electric dipole selection rules hold for describing a given point charge induced electronic excitation is presented. The point charge induced transition rates for particular electronic excitations from linear response TD-DFT were validated through comparison to excited state populations from real time TD-DFT simulations following an impulsive
\end{abstract}


point charge perturbation, then evaluated on a three-dimensional grid to map their spatial dependence for a small polybenzoid. This method, when combined with information about excited state energy gradients, represents a first step toward an $a b$ initio framework for probing the structural response of materials under electron beam irradiation due to inelastic scattering.

\section{Introduction}

Most of the widely-applied $a b$ initio approaches for the calculation/prediction of observables measured by optical spectroscopies fall under the umbrella of time dependent electronic structure theories (TD-EST). Electronic excitation energies and associated transition probabilities probed by linear absorption spectroscopy can be accessed in TD-EST formalisms either through direct simulation of the electronic dynamics in the time domain, ${ }^{1-8}$ or through application of response theory in the frequency domain. ${ }^{9-16}$ Time-dependent self consistent field (TD-SCF) ${ }^{17}$ methods, which include both time-dependent Hartree-Fock (TD-HF) and Kohn-Sham density functional theory (TD-DFT), are frequently employed in this context due to their favorable scaling with respect to system size. TD-DFT in particular exhibits one of the highest accuracyto-expense ratios of all excited state electronic structure methods, and in many cases provides a tractable first principles treatment of relatively large systems of acceptable accuracy.

In the time domain, or "real time" (RT) TD-EST simulation approach, excitation energies and transition probabilities are resolved by propagating a system initially prepared in its ground state forward in time following a "kick" from an impulsive electric field. Fourier transforming the expectation values of electric/magnetic multipole operators collected during the ensuing electronic dynamics gives access to the corresponding frequency-dependent polarizability tensors, the traces of which are proportional to rotationally-averaged cross sections for various photophysical processes. ${ }^{1,18-20}$ These same quantities can also be solved for directly to first order in the strength of a 
monochromatic time-dependent perturbation through linear response theory. ${ }^{16,21,22}$

Given the long-standing pervasiveness of TD-EST methods for modeling optical spectroscopies, it is somewhat unexpected that their use to treat the electronic excitations induced by nearby charged particles has garnered only modest attention. Historically, the theoretical treatment of charged particles incident upon materials has most commonly been formulated from the vantage of the incident particle as a scattering problem in the momentum representation. ${ }^{23-28}$ Modern first principles treatments of the scattering of incident charged particles by materials include multiple scattering Green's function approaches wherein the material's electronic structure is approximated at varying levels of sophistication, ${ }^{29-33}$ as well as methods couched in the kinematic treatment of the scattering. ${ }^{34,35}$ However, Tsubonoya, Hu, and Watanabe have recently reported the first simulations of low-energy electron wave packet diffraction by graphene nanoflakes, in which the incident electron wave packet and material's electronic degrees of freedom were co-propagated within a real time, real space TD-DFT framework. ${ }^{36}$

A common application of computational methods for modeling the interactions between materials and swift charged particles is the simulation of electron energy loss spectra. In electron energy loss spectroscopy (EELS) experiments, the electronic structure of a material is probed by analyzing the distribution of kinetic energies lost by individual electrons from an electron beam by way of their interaction with the material. Most often, only beam electrons that are scattered by the material through very small semi-angles are collected in EELS experiments. ${ }^{37}$ When only these small momentum-transfer scattering events are measured, the selection rules for electronic transitions within the material are typically consistent with those for optical excitation in the long-wavelength limit that emerge from the electric dipole approximation. ${ }^{38}$ Computational methods geared toward the simulation of EELS spectra generally treat the interactions between materials and beam electrons at this same level of approximation.

Of course, when a material interacts with a very distant charged particle, it ex- 
periences an electric field that is effectively homogenous over its entire volume (see Figure 1 for a graphical sketch of this statement). In the limit where the material and the charged particle approach infinite separation, the interaction between the external point charge and the material is completely captured by just the lowest order (dipole) term in its multipole expansion. Otherwise, the charged particles comprising the material can be close enough to the external particle to appreciate the point source nature of its associated electric field, and experience spatially inhomogenous forces that are not solely described by the dipole term. ${ }^{39}$ So, outside of the regime where the electron beam is focused far away from the material whose electronic structure it is interrogating (i.e. the "aloof" beam geometry ${ }^{40,41}$ ), some of the beam electrons incident on the material are inelastically scattered through larger angles. While the electric dipole approximation may suffice for the calculation of EELS spectra, a proper accounting of the larger angle inelastic scattering events that promote electronic transitions disallowed by electric dipole selection rules is mandatory to a complete description of the electronic response of materials perturbed by the impact of charged particles. EELS experiments which also measure beam electrons scattered (through non-dipole interactions) into larger angles, while less common, have also been reported. ${ }^{42,43}$

Accurate $a b$ initio approaches for modeling the electronic response of materials to point-source electric fields are needed to provide insight into the mechanisms of radiolytic damage sustained by materials during electron microscopy and spectroscopy experiments. In this study, we present a tractable first principles method based on TDDFT for resolving the distribution of excited electronic states populated in a material following brief exposure to an external point charge. We apply both real time and linear response TD-DFT to model the response of some simple low-Z materials (an isolated carbide ion $\left(\mathrm{C}^{4-}\right)$, benzene $\left(\mathrm{C}_{6} \mathrm{H}_{6}\right)$, and pyrene $\left.\left(\mathrm{C}_{16} \mathrm{H}_{10}\right)\right)$ to a point charge perturbation. The full scalar potential of this point charge is included in the electronic Hamiltonian to relieve any need for multipolar expansions of the electrostatic potential. This is done in the same spirit as pioneering work from List et. al., where oscillator strengths for optical transitions were calculated using the full semiclassical matter-field 


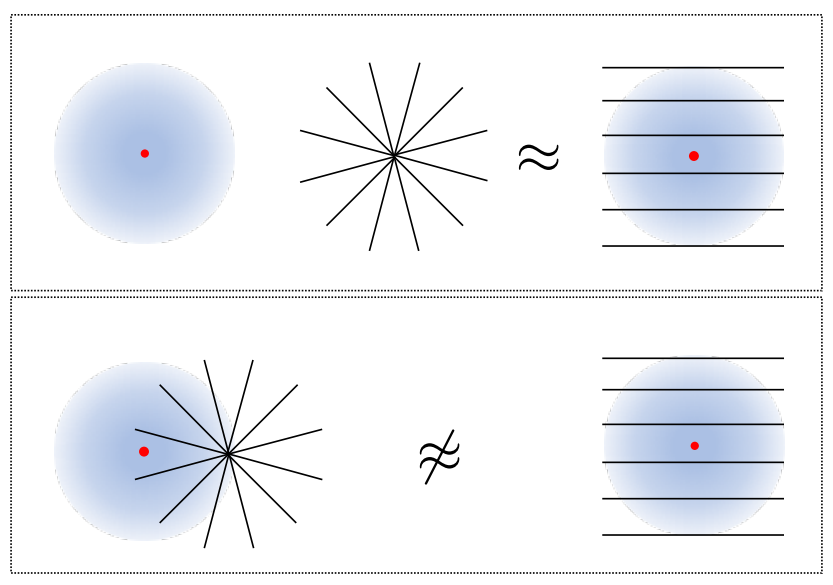

Figure 1. A sketch showing the qualitative range of validity of the electric dipole description of the interaction between an atom and an external point charge. Electric field lines are indicated in black, and negative(positive) atomic charge density in blue(red). Top panel illustrates the electric field from a distant point charge, which is essentially homogeneously dipolar over the volume of the atom. Bottom panel illustrates the inhomogenous electric field experienced by an atom when a point charge is placed near to its nucleus.

interaction operator without resorting to multipolar expansion. ${ }^{44,45}$

The remainder of this paper is structured as follows. We first describe an approximate method of accounting for beam electrons in the electronic Hamiltonian. We then provide an overview of the theoretical formalism on which the developed computational methods are built, and give the working equations for RT and LR TD-DFT. Calculations of the carbide ion and benzene molecule are then presented, which demonstrate when contributions to point charge-induced electronic transition rates from terms beyond first order in the multipolar expansion become important. We then present a general method to determine the range of separation distances over which an external point charge produces an electric field with effectively homogeneous polarization over the volume of a nearby material. Finally, to showcase the practical utility of the methodology, we present point charge position-dependent electronic excitation rates for a small polycyclic aromatic hydrocarbon, and conclude with perspective on future directions for the TD-EST description of electron beam induced excitations and structural modifications of materials. 


\section{Methodology}

All calculations were performed in a locally modified version of NWChem. ${ }^{46,47}$ In addition to the LR and RT TD-DFT calculations of electronic excitations induced by the presence of point charges, NWChem was also used (unmodified) to optimize molecular geometries, calculate LR-TD-DFT excited state energy gradients, ${ }^{48}$ and produce realspace electron density data for ground and excited states. ${ }^{46}$ Atomic units (AU) have been used throughout this paper unless otherwise noted.

\section{Impulse Approximation Description of Relativistic Elec-} trons

Because electrons in all but the heaviest atoms exhibit velocities that are a small fraction of the speed of light, the scalar (electric) potential dominates in the interaction between external electromagnetic fields and most atoms, molecules, and condensedphase materials. As such, it is common to omit the vector (magnetic) potential from the matter-field interaction Hamiltonian. The Lorentz invariant expression for the scalar potential of a charged particle translating at constant velocity is given in the retarded timeframe by the Liénard-Wiechert potential. ${ }^{49}$ In the zero velocity limit, the LiénardWiechert scalar potential reduces to the classical electrostatic (Coulomb) potential. For charged particles translating at speeds approaching that of light, however, the matter-beam interactions become shorter in duration than the electrostatic treatment would suggest due to the reduction of the the electric field intensity emanated by the

moving charge along its direction of propagation. ${ }^{50}$ In the ultra relativistic limit, a moving point charge's associated electric field is nonzero only in the plane transverse to its velocity. In this case, only the portion of a charge distribution that lies in this plane is perturbed by the fast point charge at any given instant. 


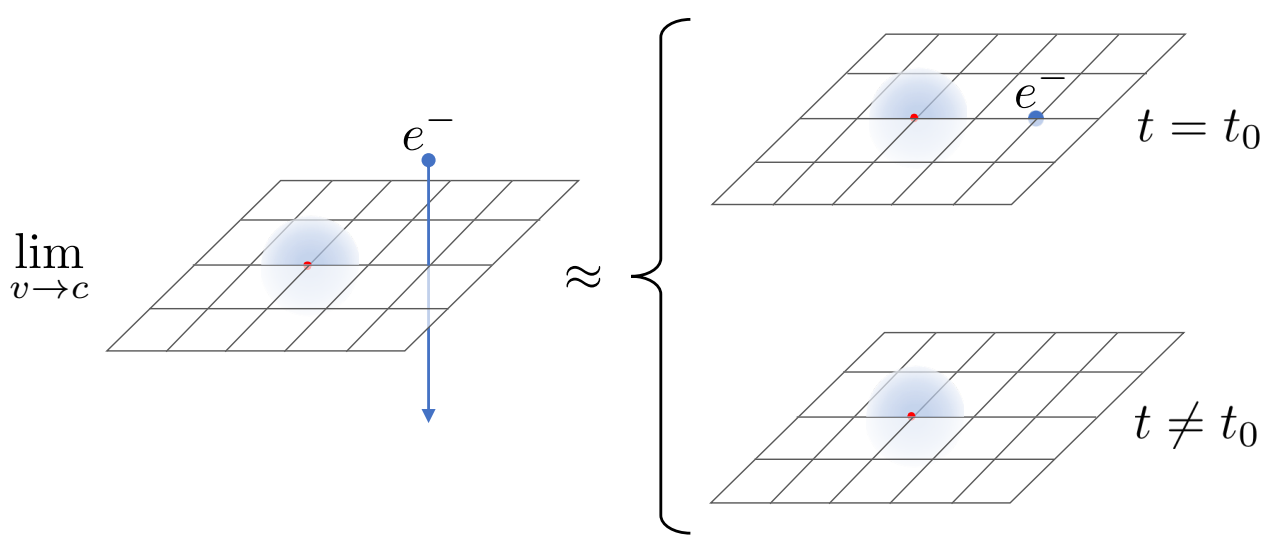

Figure 2. Schematic showing the electrostatic impulse approximation to the electric potential of an ultrarelativistic electron utilized in the current study. A piecewise description of the scalar potential of a swift electron is adopted, in which the system experiences an impulsive electrostatic potential only at the time that the point charge would be passing through the plane containing the atomic center(s).

In this preliminary study, we will only consider the interactions of isolated atoms and planar molecules with swift electrons. For time-domain simulations of the response of these "flat" systems to the passage of an ultrarelativistic electron, the scalar potential can be qualitatively approximated by an electrostatic potential impulse activated at the instant that the beam electron enters the plane containing the atomic center(s) (Fig. 2). While this may be a crude approximation to the true potential experienced by a material subjected to a finite velocity beam, it is only under this treatment that the perturbation is restricted to transferring population from the ground to excited states (and not between excited states.) The final state from the RT-TD-DFT simulation of a material subjected to the impulsive point charge perturbation described above can be compared directly to transition rates evaluated within first-order time dependent perturbation theory (using Fermi's golden rule and LR-TD-DFT.)

The operator, $\hat{V}$, for the electrostatic potential energy of a material exposed to a particle with charge $q$ located at position $\mathbf{r}_{\mathrm{pc}}$ is given by:

$$
\hat{V}\left(q, \mathbf{r}_{\mathrm{pc}}\right)=\frac{q}{\left|\hat{r}-\mathbf{r}_{\mathrm{pc}}\right|},
$$

where $\hat{r}$ is the position operator in the space of the material's electronic coordinates. 
Conveniently, $\hat{V}\left(q, \mathbf{r}_{\mathrm{pc}}\right)$ is already present in the electronic Hamiltonian to account for the electron-nuclear attraction potential, so its matrix elements are immediately available to be used for our purposes here with no additional software development effort.

In an EELS experiment, each beam electron performs two essential tasks: it perturbs the material, and it reports on the final state of the material through its energy loss. In contrast, the state of the material is known at all times during the RT-TD-DFT simulations. This allows for a material-centric perspective of the inelastic scattering process to be adopted, where only the state of the material following its perturbation by an external point charge is considered. Disregard for the final state of the scattered electron motivates the choice to express the system's electronic state in a basis of spatially-localized (Gaussian) functions, rather than the delocalized functions (e.g. plane waves) that would provide a more natural basis for the scattering states of the beam electron. The electronic states of molecular species and materials which lack long-range structural periodicity (e.g. nanoscaled, amorphous, polycrystalline or otherwise disordered materials) is most quickly-convergent when expressed in a spatially localized basis, and even crystals idealized as infinitely-extended periodic lattices will see their periodicity destroyed by the application of a point-source electric field.

\section{The TD-DFT Formalism}

As TD-DFT is now a well established method, we only provide a brief overview of the formalism and necessary working equations here and refer readers to references 51 and 13 for a complete derivation and review of the method. In the Kohn-Sham (KS) density functional theory, the many-body Schrödinger equation is recast as an effective one-electron problem for a fictitious system of non-interacting electrons (described by KS orbitals, $\left.\left\{\phi_{i}\right\}\right)$ evolving under an external potential that is designed to produce the density $(\rho(\mathbf{r}))$ of the fully-interacting system from that of the non-interacting one. ${ }^{52,53}$

The equation of motion for the KS orbitals is the time-dependent Kohn-Sham equation: ${ }^{17,54}$ 


$$
i \frac{\partial \phi_{i}(t)}{\partial t}=\hat{F} \phi_{i}(t)
$$

The KS operator, $\hat{F}$, is:

$$
\hat{F}=\hat{h}+\int d \mathbf{r}^{\prime} \frac{\rho\left(\mathbf{r}^{\prime}\right)}{\left|\mathbf{r}-\mathbf{r}^{\prime}\right|}+\frac{\delta E_{\mathrm{xc}}[\rho(\mathbf{r})]}{\delta \rho(\mathbf{r})}
$$

where:

$$
\hat{h}=-\frac{1}{2} \nabla^{2}-\sum_{k=1}^{m} \frac{Z_{k}}{\left|\mathbf{r}-\mathbf{R}_{k}\right|}
$$

and $\mathrm{m}$ is the number of nuclei in the system, $\mathbf{R}_{k}$ and $Z_{k}$ are the coordinates and charge of the $k^{t h}$ nucleus, $\mathbf{r}$ are electronic coordinates, and $E_{\mathrm{xc}}$ is the exchange-correlation energy functional. In the spin-restricted treatment for closed-shell systems, the total density is given by:

$$
\rho(\mathbf{r})=2 \sum_{i}^{o c c .}\left|\phi_{i}(\mathbf{r})\right|^{2}
$$

, where the summation in Eq. (5) runs over all doubly-occupied KS orbitals. The KS orbitals can be expanded in a finite basis of contracted, atom-centered Gaussian functions, $\left\{\chi_{\mu}\right\}$.

$$
\phi_{i}=\sum_{\mu} C_{\mu i}(t) \chi_{\mu}
$$

Henceforth, Greek characters $(\mu, \nu, \lambda, \sigma)$ will index these basis functions, lowercase Roman characters $(i, j / a, b)$ will index the (occupied/virtual) KS orbitals of the KSDFT ground state, and capital Roman characters $(I J)$ will index the (all electron) energy eigenstates.

The one particle reduced density matrix (1RDM) $\mathbf{P}$ can be expressed in the contracted Gaussian function basis through the orbital expansion coefficients from Eq. (6): 


$$
P_{\mu \nu}(t)=2 \sum_{i}^{o c c .} C_{\mu i}^{*}(t) C_{\nu i}(t)
$$

The KS matrix elements are given in the contracted Gaussian function basis by Eq. (8).

$$
F_{\mu \nu}(t)=h_{\mu \nu}+\sum_{\lambda \sigma} P_{\lambda \sigma}(t)(\mu \nu \mid \lambda \sigma)+v_{\mathrm{xc}, \mu \nu}
$$

where:

$$
\begin{gathered}
(\mu \nu \mid \lambda \sigma)=\int \mathbf{d r} \mathbf{d} \mathbf{r}^{\prime} \chi_{\mu}^{*}(\mathbf{r}) \chi_{\nu}(\mathbf{r}) \frac{1}{\left|\mathbf{r}-\mathbf{r}^{\prime}\right|} \chi_{\lambda}^{*}\left(\mathbf{r}^{\prime}\right) \chi_{\sigma}\left(\mathbf{r}^{\prime}\right) \\
v_{\mathrm{xc}, \mu \nu}=\int \mathbf{d} \mathbf{r} \chi_{\mu}^{*}(\mathbf{r}) \frac{\delta E_{\mathrm{xc}}}{\delta \rho(\mathbf{r})} \chi_{\nu}(\mathbf{r})
\end{gathered}
$$

The $1 \mathrm{RDM}$ and KS matrix can be re-expressed in an orthonormal basis, $\left\{\chi_{\mu}^{\prime}\right\}$, by way of the symmetric Löwdin transformation:

$$
\begin{aligned}
& \mathbf{P}^{\prime}=\mathbf{S}^{1 / 2} \mathbf{P} \mathbf{S}^{1 / 2} \\
& \mathbf{F}^{\prime}=\mathbf{S}^{-1 / 2} \mathbf{F} \mathbf{S}^{-1 / 2},
\end{aligned}
$$

where $S_{\mu \nu}=\int d \mathbf{r} \chi_{\mu}^{*}(\mathbf{r}) \chi_{\nu}(\mathbf{r})$. The TD-KS equation can be written in Liouville-Von Neumann form as an equation of motion for the 1RDM in the orthogonalized basis:

$$
i \frac{\partial \mathbf{P}^{\prime}(\mathbf{t})}{\partial t}=\left[\mathbf{F}^{\prime}(t), \mathbf{P}^{\prime}(t)\right]
$$

Expectation values of one-body operators and the electronic energy are calculated according to Eqs. (12) to (13) (where $A_{\mu \nu}$ are matrix elements of an arbitrary oneelectron operator, $\hat{A}$, in the nonorthogonal, Gaussian function basis). 


$$
\begin{gathered}
\langle\hat{A}\rangle=\sum_{\mu \nu} P_{\nu \mu} A_{\mu \nu} \\
\left\langle E_{\text {ele }}\right\rangle=\langle\hat{h}\rangle+\frac{1}{2} \sum_{\mu \nu} \sum_{\lambda \sigma} P_{\mu \nu} P_{\lambda \sigma}(\mu \nu \mid \lambda \sigma)+E_{\mathrm{xc}}[\rho(\mathbf{r})]
\end{gathered}
$$

\section{"Real Time" TD-DFT}

The general solution to Equation (11) is given by:

$$
\mathbf{P}^{\prime}(t)=\mathbf{U}\left(t_{0}, t\right) \mathbf{P}^{\prime}\left(t_{0}\right) \mathbf{U}^{\dagger}\left(t_{0}, t\right)
$$

Evaluation of the matrix representation of the time evolution operator, $\mathbf{U}$, requires time-ordered exponentiation (denoted in Eq. (15) by $\mathcal{T} \exp$ ) of the KS matrix integrated over the interval $\left(t_{0}, t\right)$.

$$
\mathbf{U}\left(t_{0}, t\right)=\mathcal{T} \exp \left(-i \int_{t_{0}}^{t} d t^{\prime} \mathbf{F}\left(t^{\prime}\right)\right)
$$

In this work, Eq. (15) will be approximated by the Magnus expansion thereof, truncated at second order. ${ }^{55,56}$ The KS matrix carries time dependence even in the absence of any external perturbation through its dependence on the 1RDM. However, the KS matrix may also be explicitly time-dependent through the addition of an external potential, $\hat{V}^{\text {ext }}$, to the KS operator defined in Eq. (3). In Eq. (16), both modes of time dependence are indicated by the notation $\mathbf{F}\left(\mathbf{V}^{\operatorname{ext}}(t), \mathbf{P}(t)\right)$.

$$
\begin{gathered}
\mathbf{U}\left(t, t+\Delta_{t}\right)= \\
\exp \left(\frac{-i \Delta_{t}}{2}\left(\mathbf{F}\left(\mathbf{V}^{\operatorname{ext}}(t), \mathbf{P}(t)\right)+\mathbf{F}\left(\mathbf{V}^{\operatorname{ext}}\left(t+\Delta_{t}\right), e^{-i \Delta_{t} \mathbf{F}\left(\mathbf{V}^{\text {ext }}(t), \mathbf{P}(t)\right)} \mathbf{P}(t) e^{i \Delta_{t} \mathbf{F}\left(\mathbf{V}^{\text {ext }}(t), \mathbf{P}(t)\right)}\right)\right)\right) \\
+\mathcal{O}\left(\Delta_{t}^{3}\right)
\end{gathered}
$$

The cost of a RT-TD-DFT time step is essentially equivalent to that of two iterations 
of the self consistent field method when Eq. (16) is employed to integrate the electronic Schrödinger equation. The population of the $I^{\text {th }}$ energy eigenstate, $\rho_{I}$, can be accessed indirectly through autocorrelation functions of the expectation value of observables ${ }^{57-59}$ (e.g. dipole moment) collected during the simulation according to the relation shown in Eq. (17) (See Appendix 1).

$$
\mathfrak{F}[\langle\mu(0) \mu(t)\rangle](\omega)=\sum_{I J} \rho_{I}\left|\left\langle\Psi_{I}|\hat{\mu}| \Psi_{J}\right\rangle\right|^{2} \delta\left(\omega-\omega_{I J}\right)
$$

\section{Linear Response TD-DFT}

Starting from Eq. (11) and solving for the response of a system initially in its ground state to (first order in the strength of) a monochromatic perturbation yields a nonHermitian eigenvalue problem known as the linear response TD-DFT equation, whose solutions are excitation energies, $\left\{\omega_{I}\right\}$, and one-particle transition densities $\left\{\mathbf{X}^{I}, \mathbf{Y}^{I}\right\}$ referenced to the SCF ground state: ${ }^{13,21,60-63}$

$$
\begin{gathered}
{\left[\begin{array}{cc}
\mathbf{A} & \mathbf{B} \\
\mathbf{B}^{*} & \mathbf{A}^{*}
\end{array}\right]\left[\begin{array}{l}
\mathbf{X} \\
\mathbf{Y}
\end{array}\right]=\boldsymbol{\omega}\left[\begin{array}{cc}
\mathbf{I} & \mathbf{0} \\
\mathbf{0} & -\mathbf{I}
\end{array}\right]\left[\begin{array}{l}
\mathbf{X} \\
\mathbf{Y}
\end{array}\right]} \\
A_{i a, j b}=\delta_{i j} \delta_{a b}\left(F_{a a}-F_{i i}\right)+(i a \mid j b)+\left(i a\left|f_{\mathrm{xc}}\right| j b\right) \\
B_{i a, j b}=(i a \mid b j)+\left(i a\left|f_{\mathrm{xc}}\right| b j\right),
\end{gathered}
$$

, where:

$$
\left(i a\left|f_{\mathrm{xc}}\right| j b\right)=\int \mathbf{d} \mathbf{r} \mathbf{d} \mathbf{r}^{\prime} \phi_{i}^{*}(\mathbf{r}) \phi_{a}(\mathbf{r}) \frac{\delta^{2} E_{\mathrm{xc}}}{\delta \rho(\mathbf{r}) \delta \rho\left(\mathbf{r}^{\prime}\right)} \phi_{b}^{*}\left(\mathbf{r}^{\prime}\right) \phi_{j}\left(\mathbf{r}^{\prime}\right)
$$

, and the KS matrix and two electron integrals have been transformed from the basis of non-orthogonal Gaussian functions to the KS orbital basis using the orbital coefficient matrix for the converged SCF ground state, $\mathbf{C}^{0}$ (Eq. (20)). 


$$
\begin{aligned}
(i a \mid j b) & =\sum_{\mu \nu \lambda \sigma} C_{\mu i}^{0} C_{\nu a}^{0} C_{\lambda j}^{0} C_{\sigma b}^{0}(\mu \nu \mid \lambda \sigma) \\
F_{i i} & =\sum_{\mu \nu} C_{\mu i}^{0 *} F_{\mu \nu} C_{\nu i}^{0}
\end{aligned}
$$

The ground to excited state transition moments for an arbitrary one-body operator can be evaluated in the LR-TD-SCF formalism according to Eq. (21). ${ }^{22,61,64}$ For the coupling between ground and excited states induced by an external point charge, the relevant operator is $\hat{V}$ from Eq. (1).

$$
\left\langle\Psi_{0}\left|\hat{V}\left(\mathbf{r}_{\mathrm{pc}}\right)\right| \Psi_{I}\right\rangle=\sum_{i a} V_{i a}\left(\mathbf{r}_{\mathrm{pc}}\right) X_{i a}^{I}+V_{a i}\left(\mathbf{r}_{\mathrm{pc}}\right) Y_{i a}^{I}
$$

In order to map out the the dependence of the transition potentials between electronic energy eigenstates on the point charge position, $\mathbf{r}_{\mathrm{pc}}$, one only needs to form $\mathbf{V}\left(\mathbf{r}_{\mathrm{pc}}\right)$ for different $\mathbf{r}_{\mathrm{pc}}$ and recompute its trace with the transition densities for each transition of interest according to Eq. (21).

The point charge induced transition rates between the ground and excited states can be approximated by application of the (state-to-state) Fermi's golden rule expression ${ }^{65}$ given in Eq. (22).

$$
w_{0 I}\left(\mathbf{r}_{\mathrm{pc}}\right)=2 \pi\left|\left\langle\Psi_{0}\left|\hat{V}\left(\mathbf{r}_{\mathrm{pc}}\right)\right| \Psi_{I}\right\rangle\right|^{2}
$$

\section{Results}

In each TD-SCF calculation reported in this study, the atomic or molecular system was initialized in its optimized DFT ground state. For RT-TD-DFT simulations, the TD-KS equation was integrated according to Eq. (16) with a 0.05 AU time step for a total of 50,000 steps, yielding 2,500 AU of simulated electronic dynamics. With these simulation parameters, the electronic energy was conserved to within $10^{-11}$ AU during the perturbation-free evolution. Impulsive point charge perturbations were applied as 
described in the methodology section (see Fig. 2) during only the first time step of each simulation. All spin-allowed excitations ranging from valence to core transitions were allowed (i.e. no frozen core approximation was employed.) For molecular systems, geometries were optimized in the electronic ground state at the same level of theory employed in the LR/RT TD-DFT calculations.

In addition to performing time domain simulations of the impulsively-perturbed systems, the matrix elements of the point charge potential between the DFT ground state and LR-TD-DFT excited states were also evaluated to calculate Fermi's golden rule electronic transition rates.

\section{Dipole Approximation Validity at Large Distances}

In three separate RT-TD-DFT/6-31g(d) simulations of the benzene molecule, an impulsive point charge perturbation was applied at a distance of $100 a_{0}$ from the molecular center of mass (COM) along each Cartesian axis, such that the resulting electric field is $0.0001 \mathrm{AU}$ in magnitude at the COM. Frequency domain autocorrelation functions were computed from the dipole moment expectation values collected at each time step of the simulations. To gauge the similarity of perturbations experienced by a system from a distant point charge and an homogeneous electric field, analogous simulations were performed in which a $0.0001 \mathrm{AU}$ homogenous electric field impulse polarized along each axis was applied to the system (in the same lab-frame as the previous simulations).

The rotational average of the (frequency domain) dipole autocorrelation function resulting from application of these two types of impulse perturbations are plotted in Fig. 3. While the same electronic excitations are promoted by the homogenous electric field and that of a distant point source, the excited state populations are not in quantitative agreement due to the inhomogeneity of the field strength from the point source over the volume of the molecule. Nevertheless, there are neither optically dark transitions that are promoted by the distant point charge nor optically bright transitions that are not enacted by the distant point charge perturbation, demonstrating that dipole selection rules for electron beam induced electronic excitation are upheld 
in the aloof beam geometry.

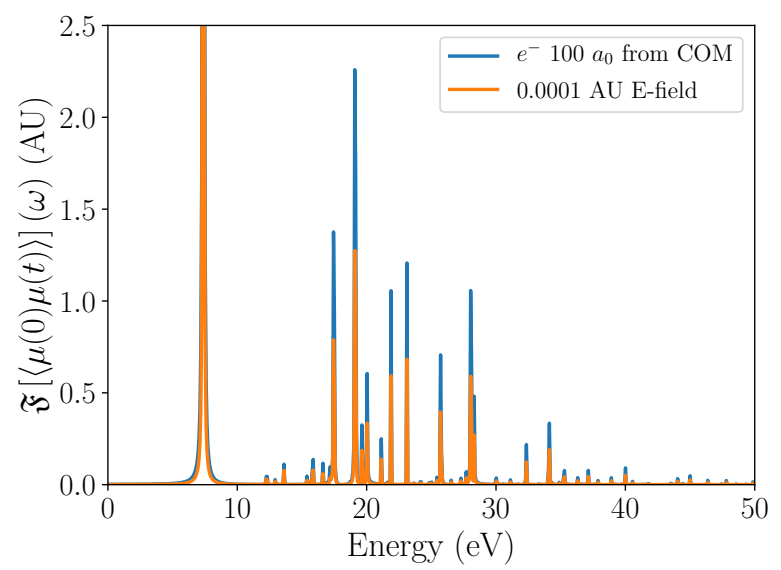

Figure 3. Rotationally-averaged, frequency domain dipole autocorrelation functions for benzene following application of a 0.0001 AU homogenous electric field impulse in $x, y$, and $z$ directions, as well as an impulsive point-source electric field from a particle of elementary charge placed $100 a_{0}$ away from benzene's center of mass (COM) along $x, y$, and $z$.

\section{Dipole Approximation Validity at Intermediate and Small}

\section{Distances}

A series of real time TD-DFT simulations were performed for an isolated carbide ion in which the impulsive point charge perturbation was applied at different distances from the carbide center of mass. The $6-31+\mathrm{g}(\mathrm{d})$ basis set was utilized, since its extra set of diffuse functions for each (valence) angular momentum shell provides an adequate description of the carbide ion's ground state electron structure, and also permits relatively large amplitude fluctuations in the electron density. The dipole autocorrelation functions reveal the changes in excited state population that result from applying the point charge perturbation at these different distances. The distribution of electronic energy eigenstates contributing to the superposition after the point source electric field perturbation is applied (see Fig. 5) varies non-monotonically in the point charge's placement.

The strongly dipole allowed (see Tab. 1), parity flipping $2 p \rightarrow 3 s$ transition shows appreciable transition rates over large distances, while the rates of parity-preserving 
transitions increase sharply with respect to the point charge's proximity to the atomic center.

The Fermi's golden rule transition rates from the ground to LR-TD-DFT excited states for particular transitions are also reported in Fig. 6 across a range of separation distances from the carbide COM. Since the perturbation is only applied during the first time step in the RT-TD-DFT simulations (and $\Delta_{t}<</\left|V_{0 I}\right|$ ) rates/probabilities from the first order perturbation theory accurately capture the weights of each excited state contributing to the final state of the system from the time-domain simulations. The peak at $\sim 2 \mathrm{eV}$ (the $2 p \rightarrow 3 s$ transition), for instance, shows close to zero transition probability from LR-TDSCF at $\sim 1 \AA$ separation and essentially zero population in the RT-TD-DFT dipole autocorrelation function. More generally, the relative excited state populations encoded into the intensity of peaks in the dipole autocorrelation functions (Eq. (17)) are proportional to the transition probabilities from LR-TD-DFT, as indicated by the near unity correlation coefficient in the linear fit of the the dipole autocorrelation function intensities and LR-TD-DFT transition rates shown in Fig. 4.

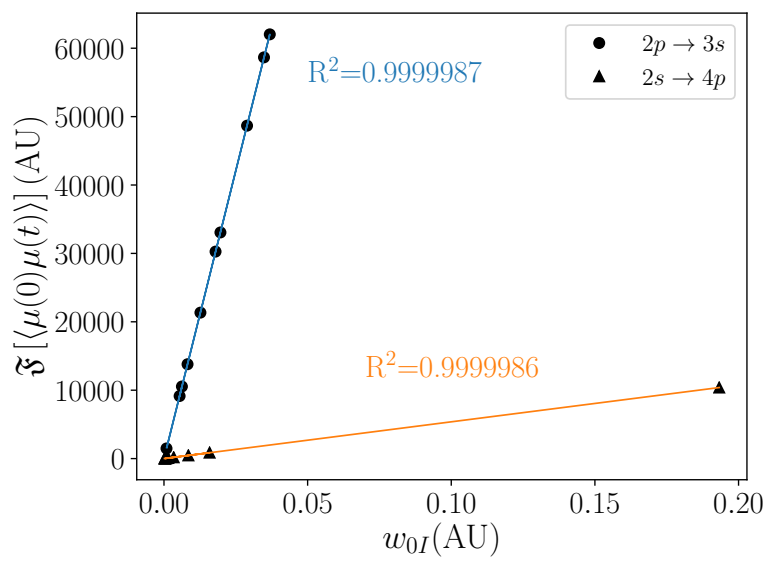

Figure 4. Plot of the dipole autocorrelation function intensities versus Fermi's golden rule transition rates evaluated over a range of distance $(0.5 \AA$ to 5.0 $\AA$ ) for the identified transitions, along with lines of best fit and corresponding coefficients of determination, $\mathrm{R}^{2}$.

To contextualize the findings of the ab initio results, consider the point charge potential matrix elements between states of well-defined parity in two extreme limits 
Table 1. Excitation energies and transition dipole strengths (mod. squared) for the dipole-allowed transitions of the carbide ion.

\begin{tabular}{|c|c|c|}
\hline Transition & Energy $(\mathrm{eV})$ & $\left\|\mu_{0 I}\right\|^{2}(\mathrm{AU})$ \\
\hline \hline $2 p \rightarrow 3 s$ & 2.3880 & 4.6093 \\
\hline $2 s \rightarrow 3 p$ & 12.147 & 0.1373 \\
\hline $2 p \rightarrow 4 s$ & 25.864 & 0.1661 \\
\hline $2 s \rightarrow 4 p$ & 29.409 & 0.1443 \\
\hline $2 p \rightarrow 3 d$ & 53.490 & 0.5178 \\
\hline $1 s \rightarrow 3 p$ & 275.66 & 0.0060 \\
\hline $1 s \rightarrow 4 p$ & 294.80 & 0.0103 \\
\hline \hline
\end{tabular}

for the point charge's position relative to the atomic center. The electric field that the atom experiences from a point charge at infinite separation distance approaches perfect spatial homogeneity, and is therefore even(gerade) in the center of mass coordinates of the atom. Conversely, a point charge residing at the atomic center of mass produces an odd(ungerade) electric field in this same coordinate system. Consequentially, both parity preserving and parity flipping transitions can be induced by the point charge perturbation depending on its location relative to the inversion center.

Take, for instance, the electrons occupying the $2 s$ orbital of carbide in its electronic ground state. When a repulsive point charge is placed in the interior of their spherically-symmetric charge distribution, the average forces they experience will be oriented outward (radially). In the limit where the negative point charge is placed directly at the atom's center, the charge contributed by one proton is negated. In this limit, the eigenstates of the perturbed electronic Hamiltonian for carbide are (neglecting hyperfine splitting) identical to the more loosely-bound states of the unperturbed boride ion. In terms of the energy eigenstates of the carbide ion, though, this corresponds to a transition to a state in which $s$ orbitals of higher principle quantum number become populated. Parity preserving transitions such as these are forbidden by symmetry under the electric dipole approximation. That they are quite strongly allowed under the full scalar potential of a perturbing charge in close proximity to the atomic center (see Fig. 6 and Fig. 7) indicates that terms beyond the dipole in the multipolar expansion are required to recover the nonzero rates for these transitions. 
The disagreement between transition dipole strength and EELS intensity for systems exhibiting inversion symmetry has been appreciated experimentally in the case of electron beam excitation of optically-dark localized surface plasmon resonances in metal nanoparticles ${ }^{66}$ and the hybridized modes of their dimers. ${ }^{67,68}$
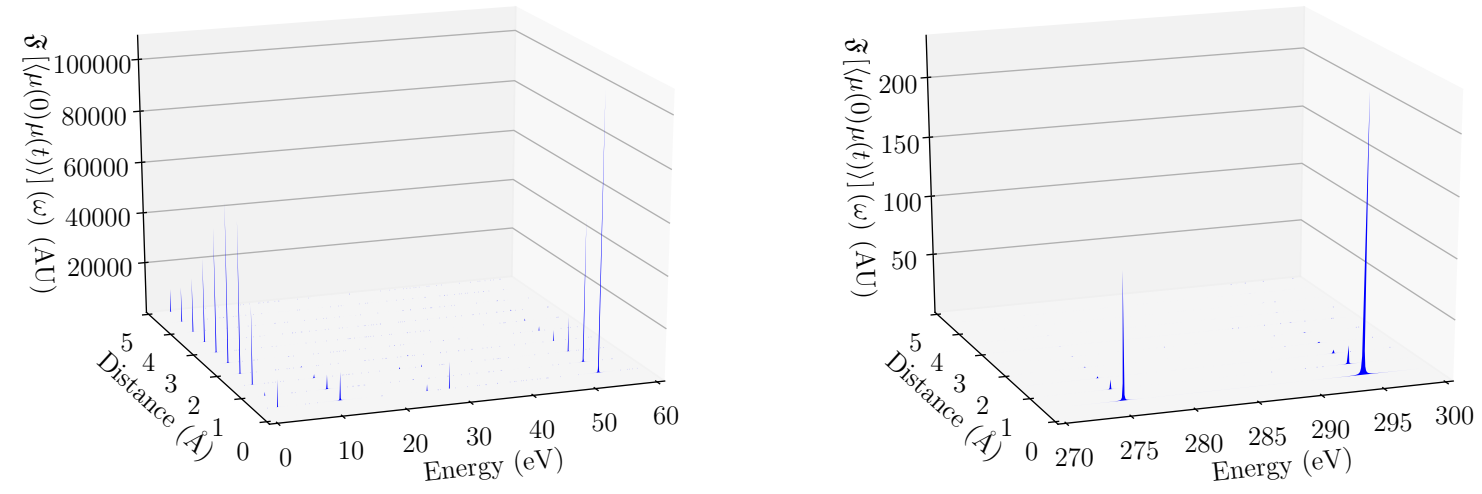

Figure 5. (left) Valence excitation region of the frequency-domain dipole autocorrelation function, $\mathfrak{F}[\langle\mu(0) \mu(t)\rangle](\omega)$ for separation distances between the carbide COM and point charge ranging from 0.5 to 5 Ångstroms. (right) Carbon k-edge region of the spectrum with differently scaled axes relative to valence region on left for clarity of presentation.

To summarize this section, we have shown that the proportionality between a point charge induced electronic excitation's probability and its corresponding transition dipole strength is broken when the distance between the external point charge and the material's bound electron density is small, showing zero dipole strength for transitions that are significantly allowed under the full electric potential of the point charge for certain positions (and vice versa.) This is the Fourier compliment to the well-known caveat that the electric dipole approximation is valid only in the small momentum transfer limit. ${ }^{39}$ However, since the position of a convergent electron beam can now routinely be controlled with sub-Ångstrom resolution in modern aberration corrected scanning transmission electron microscopes, methods for determining the allowedness of electronic transitions for a given beam position may prove to be of considerable practical value. 


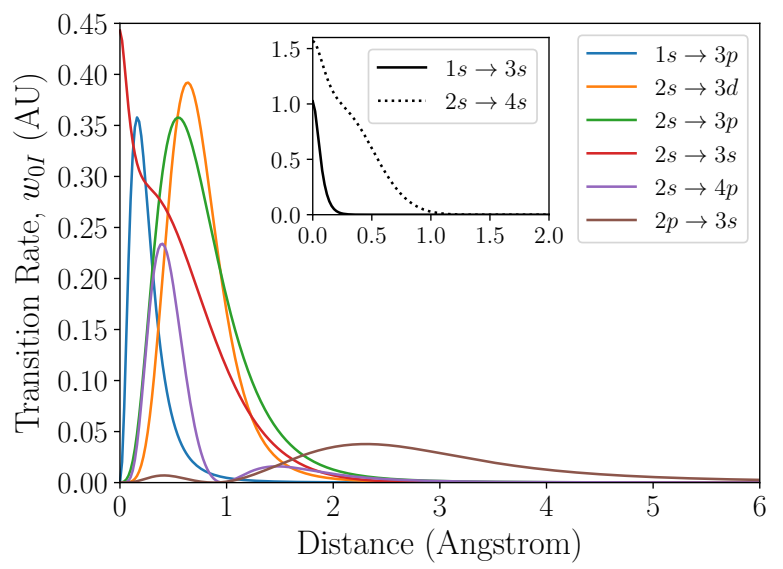

Figure 6. Fermi's golden rule rates for representative transitions, $w_{0 I}$, calculated according to Eq. (22) for the isolated carbide ion across a range of impact parameters. For transitions involving $p$ and $d$ orbitals, the reported rates are the summed rates for all degenerate excitations.

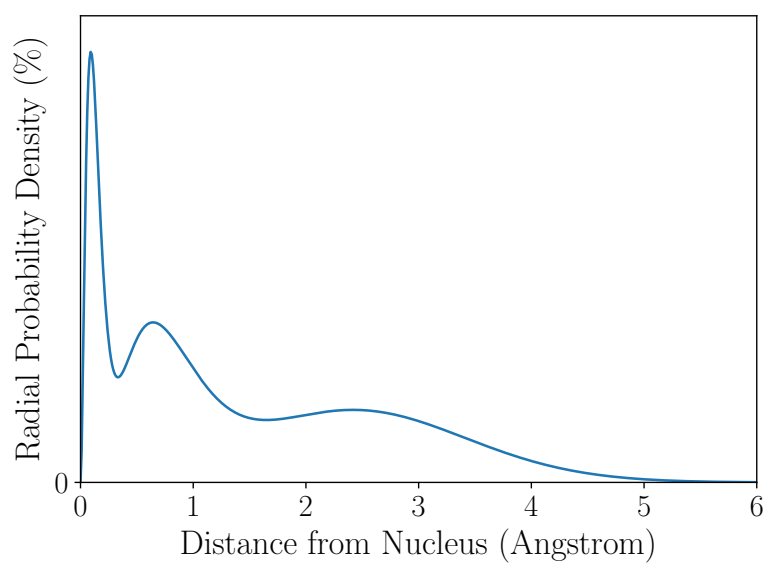

Figure 7. Ground state radial probability density for carbide calculated at the B3LYP $/ 6-31+\mathrm{g}(\mathrm{d})$ level of theory. 


\section{Dipole Approximation Validity and Point Charge Proxim-}

ity

A charged particle emanates an electric field with magnitude proportional to the inverse squared distance from the particle, so it is straight-forward to renormalize the transition rates, $w_{0 I}$, at a given impact parameter with respect to the electric field strength. In this way, the dependence of electronic transition rates on the degree of the electric field's polarization inhomogeneity can be isolated. Rates that have been renormalized with respect to the electric field intensity are plotted in Fig. 8 for representative transitions. Since the polarization of the electric field from a point source approaches homogeneity at large distances from the point source, the impact parameter at which a renormalized transition rate in Fig. 8 converges to its long distance asymptotic value indicates the critical distance where the electric field from the point charge becomes effectively homogenous over the volume of the transition density. For point charges positioned this distance or further from the material, the dipole description of the field polarization holds exactly. Optically bright transitions in Fig. 8 are distinguished from the dipole-disallowed transitions by their non-zero renormalized rates in the large impact parameter limit. For the dipole-forbidden, but quadrupole-allowed $2 s \rightarrow 3 d$ transitions, the non-convergence of the renormalized rates at large impact parameters is consistent with a priori knowledge of the dipole approximation's inadequacy over all impact parameters.

The range of impact parameters over which the dipole approximation holds for a particular electronic excitation is ultimately determined by the spatial extent of the corresponding transition density. A cursory visual comparison of the determined dipole approximation validity range and the radial electron probability density (Fig. 7) suggests a general rule-of-thumb though: terms beyond first order in the multipolar expansion of the electronic transition probability can be non-negligible whenever a perturbing point charge is placed into a region where a material's electron density is substantially nonzero. 


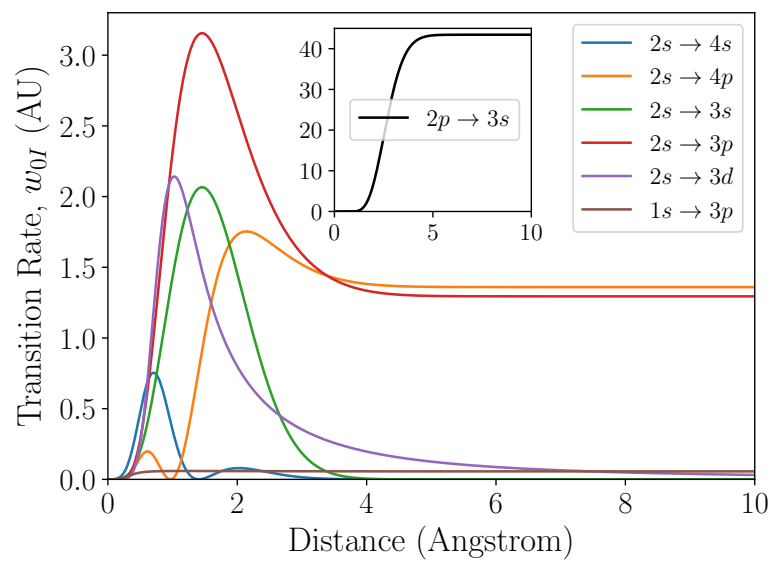

Figure 8. Fermi's golden rule transition rates, $w_{0 I}$, calculated with coupling matrix elements that have been multiplied by the square of the distance (from the atomic $\mathrm{COM}$ ) at which they are calculated to renormalize them with respect to the corresponding electric field strength.

\section{Point Charge Induced Electronic Excitation Maps}

By evaluating the electronic transition rates for different point charge locations in and around the volume of a material, the point charge positions which maximize a given transition rate can be identified. We demonstrate this here for the pyrene molecule described by the B3LYP/6-31g(d) model chemistry. The position-dependent transition rates, $w_{0 I}$ (Eq. (22)), were evaluated on a regularly-spaced grid with 0.1 Åresolution, and are plotted as isosurfaces in Fig. 9. The position dependent breakdown of the dipole selection rules is again demonstrated for this molecular system, with transitions to excited states exhibiting zero transition dipole strength from the ground state becoming strongly allowed for certain point charge positions.

One may suspect that for a given transition, regions in which electron density is depleted in the excited state (i.e. negative difference density relative to the ground state) would correspond to the regions where the probability of point charge induced transition would be large. After all, the effect of the negative point charge is to repel the like charged electron density. The difference densities (relaxed ${ }^{69}$ excited state density minus the ground state density) of the three transitions for which the position- 

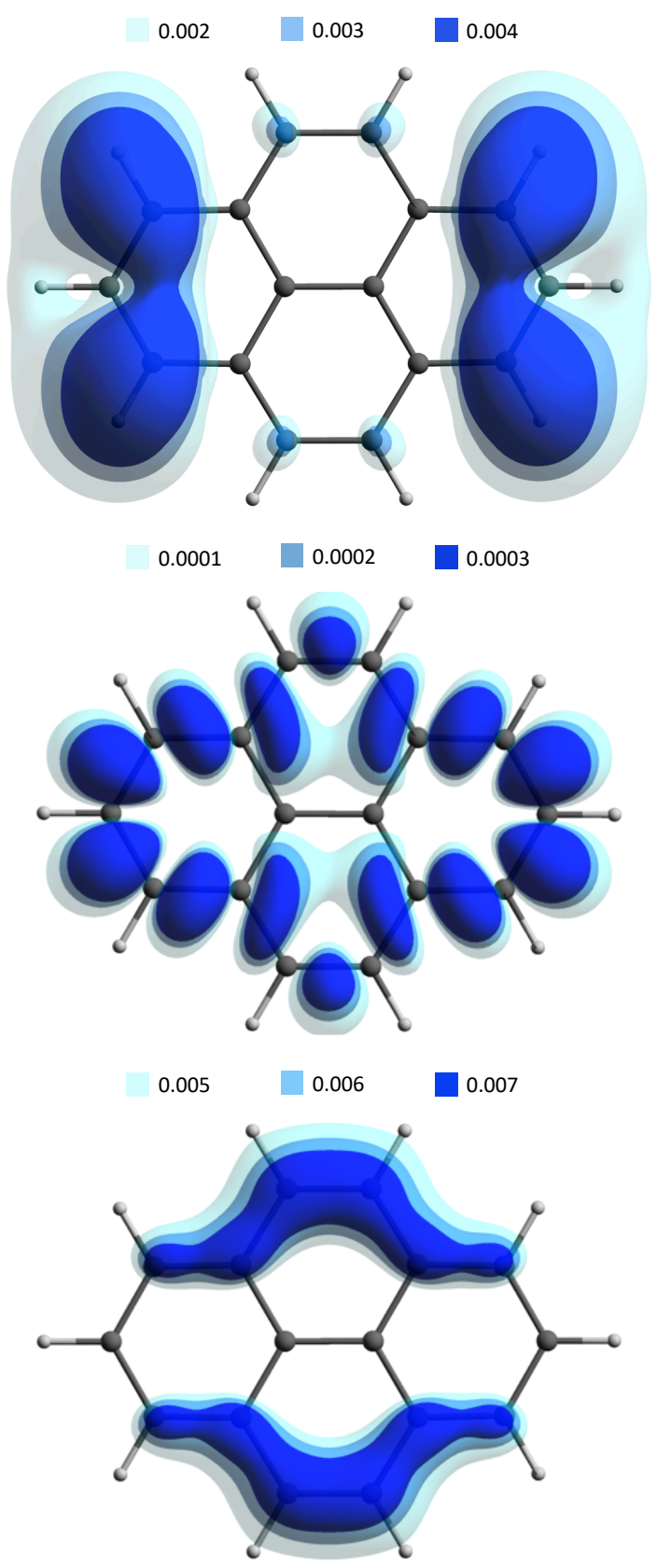

Figure 9. Spatial dependence of the transition rate from the ground to three lowest energy excited singlet states of pyrene. From top to bottom: transition rate $\left(w_{0 I}\right)$ isosurfaces for the ground to first $\left(3.72 \mathrm{eV},\left\|\mu_{01}\right\|=2.8\right)$, second $(3.79 \mathrm{eV}$, $\left.\left\|\mu_{02}\right\|=3.5 \times 10^{-4}\right)$, and third $\left(6.46 \mathrm{eV},\left\|\mu_{03}\right\|=1 . \times 10^{-10}\right)$ singlet excited states, with isovalues indicated by color in AU. 
dependent point charge transition rates are plotted in Fig. 9 were calculated, and are shown as isosurfaces in Fig. $10 .{ }^{70}$ Owing to the fact that electron density that is repelled by the beam must also be displaced in the direction of regions of positive difference density for that same transition for strong coupling, the difference densities do not show any appreciable correlation with the transition rate isosurfaces in Fig. 9.

Finally, we have also evaluated the excited state energy gradients (at the ground state equilibrium geometry) in the three lowest energy excited states of pyrene. These excited state forces determine the structural response of the material in the instants following the electronic excitation. With the combined knowledge of excited state forces and the spatial dependence of point charge induced electronic excitation probabilities, one can begin to determine where an electron beam should be focused in order to activate a particular local vibration through inelastic scattering. In the event that the forces in the excited state are sufficient to overcome energetic barriers preventing rearrangement/isomerization, electronic excitation by beam electrons can provide a route to selectively manipulate the structure of materials. Such beam-induced isomerization events have been observed during transmission electron microscopy imaging of graphene with beam energies well below the knock-on threshold, ${ }^{71}$ suggesting a nontrivial role for electronic excitations in facilitating the isomerization. While the role of excited state vibrational evolution and nonadiabatic relaxation through conical intersections in the photoisomerization of molecular systems ${ }^{72,73}$ (and photoinduced phase transitions in condensed matter ${ }^{74,75}$ ) is well recognized, the potential for harnessing these processes to induce localized structural modifications in materials through selective electron beam exposure is yet to be widely appreciated. The ability to engineer local structural modifications of materials with convergent electron beams could represent a milestone on the pathway to atomically precise materials manipulation and manufacturing technologies. 

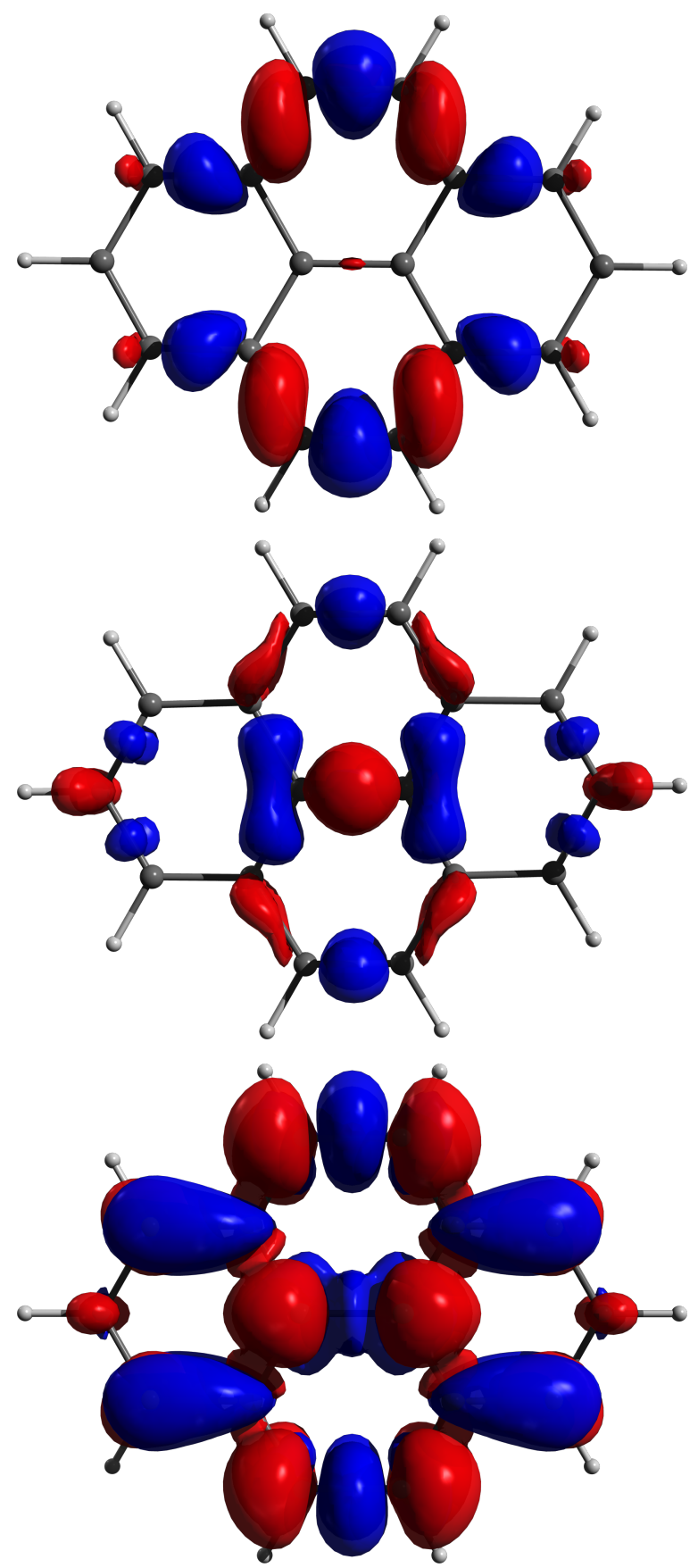

Figure 10. Charge density difference isosurfaces (isovalue $= \pm 0.001 e / a_{0}^{3}$ ) for the three lowest energy transitions of pyrene (presented in the same order as Fig. 9.) Red(blue) indicates depleted(excess) density relative to the ground state. 

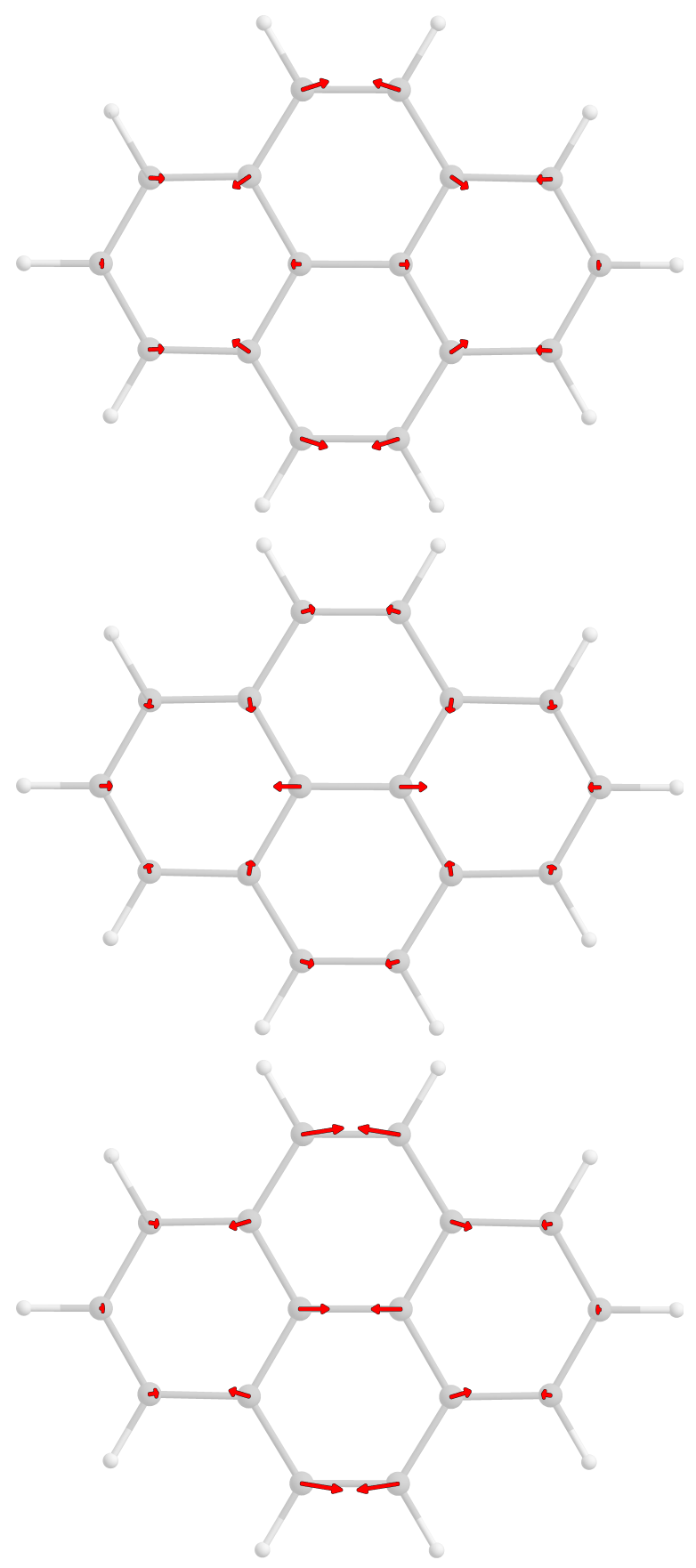

Figure 11. Changes in forces on nuclei upon electronic excitation to the three lowest energy singlet excited states in pyrene (presented in the same order as Fig. 9.) Force vectors are scaled identically for all three electronic transitions. Note that all forces lie in the plane of the molecule for these particular transitions. 


\section{Summary and Conclusions}

In this paper, methods for evaluating rates of point charge induced transitions between electronic states in materials using linear response TD-DFT were presented, and shown to be consistent with the final populations of excited electronic states from real time TD-DFT simulations following an impulsive point source electric field perturbation. We also presented a straight-forward method to determine the validity of the electric dipole approximation to the electric potential between a material and external point charge at a given spatial separation. It was shown that the dipole approximation fails to capture certain allowed transitions in materials that exhibit inversion symmetry. More generally, terms beyond the dipole in the expansion of the scalar potential are essential to a correct description of the selection rules when the perturbing charge is located near to or inside of a material's distribution of bound electron probability density. This scenario is ubiquitous in routine operation of EELS and electron microscopy instrumentation, so the methods put forth here will allow a more complete understanding of energy transfer from electron beam to the excited electronic states of materials, and therefore the structural damage that samples sustain under electron beam irradiation.

Furthermore, we've shown how the point charge induced electronic excitation rates can be used in conjunction with excited state energy gradient calculation to deduce the instantaneous vibrational response in materials to a point charge perturbation applied at specific position. This represents a humble first step toward the theory-guided engineering of number-conserving (i.e. non- "sputtering") structural transformations of materials mediated by inelastic electron scattering.

In followup studies, the Authors plan to utilize the methods detailed here to investigate the response of nanophase materials to electron beam perturbations. Extensions of the method to utilize the relativistically correct form for the scalar potential of a swift charged particle are also planned, as well as simulations of the coupled electronic and vibrational response of the material via a mixed quantum-classical dynamics 
scheme $^{76,77}$ capable of reproducing electron beam induced structural modifications of materials.

\section{Appendix A}

The autocorrelation function of an operator, $\hat{A}$, is an even function defined by:

$$
\langle\hat{A}(0) \hat{A}(t)\rangle=\lim _{t^{\prime} \rightarrow \infty} \frac{1}{t^{\prime}} \int_{0}^{t^{\prime}} d \tau \hat{A}(\tau) \hat{A}(\tau+t)
$$

Expressing the product of operators $\hat{A}(\tau) \hat{A}(\tau+t)$ in the electronic energy eigenbasis, $\left\{\Psi_{I}\right\}$, and recognizing that energy eigenstate populations $\left\{\rho_{I}\right\}$ are constants of the motion for a closed system (rendering the microcanonical ensemble average and time average equivalent) gives:

$$
\langle\hat{A}(0) \hat{A}(t)\rangle=\sum_{I} \rho_{I}\left\langle\Psi_{I}|\hat{A}(0) \hat{A}(t)| \Psi_{I}\right\rangle
$$

Resolving the identity and making use of the definition of the time evolution operator gives:

$$
\begin{aligned}
\langle\hat{A}(0) \hat{A}(t)\rangle & =\sum_{I J} \rho_{I}\left\langle\Psi_{I}|\hat{A}(0)| \Psi_{J}\right\rangle\left\langle\Psi_{J}|\hat{A}(t)| \Psi_{I}\right\rangle \\
& =\sum_{I J} \rho_{I}\left\langle\Psi_{I}|\hat{A}(0)| \Psi_{J}\right\rangle\left\langle\Psi_{J}\left|\hat{U}^{\dagger}(0, t) \hat{A}(0) \hat{U}(0, t)\right| \Psi_{I}\right\rangle
\end{aligned}
$$

Since $\left\{\Psi_{I}\right\}$ are the solutions to the time independent Schrödinger equation, $\hat{H} \Psi_{I}=$ $\Psi_{I} E_{I}$, the time evolution and (time-independent) Hamiltonian operators commute, the autocorrelation function expression simplifies to:

$$
\langle\hat{A}(0) \hat{A}(t)\rangle=\sum_{I J} \rho_{I}\left\langle\Psi_{I}|\hat{A}| \Psi_{J}\right\rangle\left\langle\Psi_{J}|\hat{A}| \Psi_{I}\right\rangle e^{-i\left(E_{I}-E_{J}\right) t}
$$

Defining $\omega_{I J}=E_{I}-E_{J}$, letting $\hat{A}=\hat{\mu}$, and Fourier transforming yields Eq. (17) directly. 


\section{References}

(1) Yabana, K.; Bertsch, G. Time-Dependent Local-Density Approximation in Real Time. Phys. Rev. B 1996, 54, 4484.

(2) Nascimento, D. R.; DePrince III, A. E. Linear Absorption Spectra from Explicitly Time-Dependent Equation-of-Motion Coupled-Cluster Theory. J. Chem. Theory Comput. 2016, 12, 5834-5840.

(3) Sonk, J. A.; Caricato, M.; Schlegel, H. B. TD-CI Simulation of the Electronic Optical Response of Molecules in Intense Fields: Comparison of RPA, CIS, CIS (D), and EOM-CCSD. J. Phys. Chem. A 2011, 115, 4678-4690.

(4) Goings, J. J.; Lestrange, P. J.; Li, X. Real-Time Time-Dependent Electronic Structure Theory. WIRES Comput. Mol. Sci. 2018, 8, e1341.

(5) Luppi, E.; Head-Gordon, M. Computation of High-Harmonic Generation Spectra of $\mathrm{H}_{2}$ and $\mathrm{N}_{2}$ in Intense Laser Pulses Using Quantum Chemistry Methods and Time-Dependent Density Functional Theory. Mol. Phys. 2012, 110, 909-923.

(6) Ghosh, S.; Andersen, A.; Gagliardi, L.; Cramer, C. J.; Govind, N. Modeling Optical Spectra of Large Organic Systems Using Real-Time Propagation of Semiempirical Effective Hamiltonians. J. Chem. Theory Comput. 2017, 13, 4410-4420.

(7) Nguyen, T. S.; Koh, J. H.; Lefelhocz, S.; Parkhill, J. Black-Box, Real-Time Simulations of Transient Absorption Spectroscopy. J. Phys. Chem. Lett. 2016, 7, $1590-1595$.

(8) Wu, X.; Teuler, J.-M.; Cailliez, F.; Clavaguera, C.; Salahub, D. R.; de la Lande, A. Simulating Electron Dynamics in Polarizable Environments. J. Chem. Theory Comput. 2017, 13, 3985-4002.

(9) Oddershede, J. Methods in computational molecular physics; Springer, 1992; pp $303-324$. 
(10) Oddershede, J. Propagator Methods. Adv. Chem. Phys. 1987, 69, 201-239.

(11) Sekino, H.; Bartlett, R. J. A Linear Response, Coupled-Cluster Theory for Excitation Energy. Int. J. Quantum Chem. 1984, 26, 255-265.

(12) Shavitt, I.; Bartlett, R. J. Many-Body Methods in Chemistry and Physics: MBPT and Coupled-Cluster Theory; Cambridge university press, 2009.

(13) Dreuw, A.; Head-Gordon, M. Single-Reference ab Initio Methods for the Calculation of Excited States of Large Molecules. Chem. Rev. 2005, 105, 4009-4037.

(14) Trofimov, A.; Krivdina, I.; Weller, J.; Schirmer, J. Algebraic-Diagrammatic Construction Propagator Approach to Molecular Response Properties. Chem. Phys, 2006, 329, 1-10.

(15) Dreuw, A.; Wormit, M. The Algebraic Diagrammatic Construction Scheme for the Polarization Propagator for the Calculation of Excited States. WIRES Comput. Mol. Sci. 2015, 5, 82-95.

(16) Olsen, J.; Jo/rgensen, P. Linear and Nonlinear Response Functions for an Exact State and for an MCSCF State. J. Chem. Phys. 1985, 82, 3235-3264.

(17) Dirac, P. A. Note on Exchange Phenomena in the Thomas Atom. Mathematical Proceedings of the Cambridge Philosophical Society. 1930; pp 376-385.

(18) Bertsch, G. F.; Iwata, J.-I.; Rubio, A.; Yabana, K. Real-Space, Real-Time Method for the Dielectric Function. Phys. Rev. B 2000, 62, 7998-8002.

(19) Goings, J. J.; Li, X. An Atomic Orbital Based Real-Time Time-Dependent Density Functional Theory for Computing Electronic Circular Dichroism Band Spectra. J. Chem. Phys. 2016, 144, 234102.

(20) Goings, J. J.; Lingerfelt, D. B.; Li, X. Can Quantized Vibrational Effects Be Obtained from Ehrenfest Mixed Quantum-Classical Dynamics? J. Phys. Chem. Lett. 2016, 7, 5193-5197. 
(21) Jørgensen, P. Second Quantization-Based Methods in Quantum Chemistry; Elsevier, 2012.

(22) Linderberg, J.; Öhrn, Y.; Sons, J. W. . Propagators in Quantum Chemistry; Theoretical chemistry; Wiley, 2004.

(23) Bethe, H. Zur Theorie des Durchgangs schneller Korpuskularstrahlen durch Materie. Ann. Phys. (Berl.) 1930, 397, 325-400.

(24) Inokuti, M.; Bederson, B. Bethe's Contributions to Atomic and Molecular Physics. Phys. Scr. 2006, 73, C98.

(25) Inokuti, M. Inelastic Collisions of Fast Charged Particles with Atoms and Molecules - the Bethe Theory Revisited. Rev. Mod. Phys. 1971, 43, 297.

(26) Leapman, R.; Rez, P.; Mayers, D. K, L, and M Shell Generalized Oscillator Strengths and Ionization Cross Sections for Fast Electron Collisions. J. Chem. Phys. 1980, 72, 1232-1243.

(27) Egerton, R. F. Electron Energy-Loss Spectroscopy in the Electron Microscope; Springer Science \& Business Media, 2011.

(28) Carron, N. J. An Introduction to the Passage of Energetic Particles Through Matter; CRC Press, 2006.

(29) Jorissen, K.; Rehr, J.; Verbeeck, J. Multiple Scattering Calculations of Relativistic Electron Energy Loss Spectra. Phys. Rev. B 2010, 81, 155108.

(30) Moreno, M.; Jorissen, K.; Rehr, J. Practical aspects of electron energy-loss spectroscopy (EELS) calculations using FEFF8. Micron 2007, 38, 1-11.

(31) Oxley, M. P.; Kapetanakis, M. D.; Prange, M. P.; Varela, M.; Pennycook, S. J.; Pantelides, S. T. Simulation of Probe Position-Dependent Electron Energy-Loss Fine Structure. Microsc. Microanal. 2014, 20, 784-797. 
(32) Oxley, M. P.; Lupini, A. R.; Pennycook, S. J. Ultra-High Resolution Electron Microscopy. Rep. Prog. Phys. 2016, 80, 026101.

(33) Kapetanakis, M. D.; Zhou, W.; Oxley, M. P.; Lee, J.; Prange, M. P.; Pennycook, S. J.; Idrobo, J. C.; Pantelides, S. T. Low-Loss Electron Energy Loss Spectroscopy: An Atomic-Resolution Complement to Optical Spectroscopies and Application to Graphene. Phys. Rev. B 2015, 92, 125147.

(34) Tait, E.; Ratcliff, L.; Payne, M. C.; Haynes, P.; Hine, N. Simulation of Electron Energy Loss Spectra of Nanomaterials with Linear-Scaling Density Functional Theory. J. Phys. Condens. Matter 2016, 28, 195202.

(35) Mizoguchi, T.; Tanaka, I.; Gao, S.-P.; Pickard, C. J. First-Principles Calculation of Spectral Features, Chemical Shift and Absolute Threshold of ELNES and XANES Using a Plane Wave Pseudopotential Method. J J. Phys. Condens. Matter 2009, 21, 104204.

(36) Tsubonoya, K.; Hu, C.; Watanabe, K. Time-Dependent Density-Functional Theory Simulation of Electron Wave-Packet Scattering with Nanoflakes. Phys. Rev. B 2014, 90, 035416 .

(37) Egerton, R. F. Electron Energy-Loss Spectroscopy in the TEM. Reports on Progress in Physics 2009, 72, 016502.

(38) Cotton, F. A. Chemical Applications of Group Theory; John Wiley \& Sons, 2003.

(39) Saldin, D. K.; Yao, J. M. Dipole Approximation in Electron-Energy-Loss Spectroscopy: K-Shell Excitations. Phys. Rev. B 1990, 41, 52-61.

(40) Howie, A. Surface Reactions and Excitations. Ultramicroscopy 1983, 11, 141 148.

(41) Rez, P.; Aoki, T.; March, K.; Gur, D.; Krivanek, O. L.; Dellby, N.; Lovejoy, T. C.; Wolf, S. G.; Cohen, H. Damage-Free Vibrational Spectroscopy of Biological Materials in the Electron Microscope. Nat. Commun. 2016, 7, 10945. 
(42) Krivanek, O.; Disko, M.; Taftø, J.; Spence, J. Electron Energy Loss Spectroscopy as a Probe of the Local Atomic Environment. Ultramicroscopy 1982, 9, 249 254.

(43) Jiang, N.; Spence, J. C. H. Core-Hole Effects on Electron Energy-Loss Spectroscopy of $\mathrm{Li}_{2} \mathrm{O}$. Phys. Rev. B 2004, 69, 115112.

(44) List, N. H.; Kauczor, J.; Saue, T.; Jensen, H. J. A.; Norman, P. Beyond the Electric-Dipole Approximation: A Formulation and Implementation of Molecular Response Theory for the Description of Absorption of Electromagnetic Field Radiation. J. Chem. Phys. 2015, 142, 244111.

(45) List, N. H.; Saue, T.; Norman, P. Rotationally Averaged Linear Absorption Spectra Beyond the Electric-Dipole Approximation. Mol. Phys. 2017, 115, 63-74.

(46) Valiev, M.; Bylaska, E.; Govind, N.; Kowalski, K.; Straatsma, T.; Dam, H. V.; Wang, D.; Nieplocha, J.; Apra, E.; Windus, T.; de Jong, W. NWChem: A Comprehensive and Scalable Open-Source Solution for Large Scale Molecular Simulations. Comput. Phys. Commun. 2010, 181, $1477-1489$.

(47) Lopata, K.; Govind, N. Modeling Fast Electron Dynamics with Real-Time TimeDependent Density Functional Theory: Application to Small Molecules and Chromophores. J. Chem. Theory Comput. 2011, 7, 1344-1355.

(48) Silverstein, D. W.; Govind, N.; van Dam, H. J. J.; Jensen, L. Simulating OnePhoton Absorption and Resonance Raman Scattering Spectra Using Analytical Excited State Energy Gradients within Time-Dependent Density Functional Theory. J. Chem. Theory Comput. 2013, 9, 5490-5503.

(49) Jackson, J. Classical Electrodynamics; Wiley, 1975.

(50) Feynman, R.; Leighton, R.; Sands, M. The Feynman Lectures on Physics: Mainly Electromagnetism and Matter; The Feynman Lectures on Physics; AddisonWesley Publishing Company, 1965. 
(51) Marques, M.; Gross, E. Time-Dependent Density Functional Theory. Annu. Rev. Phys. Chem. 2004, 55, 427-455.

(52) Hohenberg, P.; Kohn, W. Inhomogeneous Electron Gas. Phys. Rev. 1964, 136, B864.

(53) Kohn, W.; Sham, L. J. Self-Consistent Equations Including Exchange and Correlation Effects. Phys. Rev. 1965, 140, A1133.

(54) Runge, E.; Gross, E. K. U. Density-Functional Theory for Time-Dependent Systems. Phys. Rev. Lett. 1984, 52, 997-1000.

(55) Magnus, W. On the Exponential Solution of Differential Equations for a Linear Operator. Commun. Pure Appl. Math. 1954, 7, 649-673.

(56) Blanes, S.; Casas, F.; Oteo, J.; Ros, J. The Magnus Expansion and Some of its Applications. Phys. Rep. 2009, 470, 151-238.

(57) Oxtoby, D. W. Vibrational Population Relaxation in Liquids. Adv. Chem. Phys. 1981, 487-519.

(58) Egorov, S.; Everitt, K.; Skinner, J. Quantum Dynamics and Vibrational Relaxation. J. Phys. Chem. A 1999, 103, 9494-9499.

(59) Berne, B. J.; Harp, G. On the Calculation of Time Correlation Functions. Adv. Chem. Phys. 1970, 63-227.

(60) Olsen, J.; Jensen, H. J. A.; Jørgensen, P. Solution of the Large Matrix Equations Which Occur in Response Theory. J. Comput. Phys. 1988, 74, 265-282.

(61) Ring, P.; Schuck, P. The Nuclear Many-Body Problem; Physics and astronomy online library; Springer, 2004.

(62) Casida, M. E. Recent Advances In Density Functional Methods: (Part I); World Scientific, 1995; pp 155-192. 
(63) Furche, F.; Ahlrichs, R. Adiabatic Time-Dependent Density Functional Methods for Excited State Properties. J. Chem. Phys. 2002, 117, 7433-7447.

(64) Hansen, A. E.; Bouman, T. D. Hypervirial Relations as Constraints in Calculations of Electronic Excitation Properties: The Random Phase Approximation in Configuration Interaction Language. Mol. Phys. 1979, 37, 1713-1724.

(65) Schatz, G.; Ratner, M. Quantum Mechanics in Chemistry; Dover Books on Chemistry; Dover Publications, 2002.

(66) Rossouw, D.; Couillard, M.; Vickery, J.; Kumacheva, E.; Botton, G. A. Multipolar Plasmonic Resonances in Silver Nanowire Antennas Imaged with a Subnanometer Electron Probe. Nano Lett. 2011, 11, 1499-1504.

(67) Koh, A. L.; Bao, K.; Khan, I.; Smith, W. E.; Kothleitner, G.; Nordlander, P.; Maier, S. A.; McComb, D. W. Electron Energy-Loss Spectroscopy (EELS) of Surface Plasmons in Single Silver Nanoparticles and Dimers: Influence of Beam Damage and Mapping of Dark Modes. ACS nano 2009, 3, 3015-3022.

(68) Bigelow, N. W.; Vaschillo, A.; Iberi, V.; Camden, J. P.; Masiello, D. J. Characterization of the Electron-and Photon-Driven Plasmonic Excitations of Metal Nanorods. ACS nano 2012, 6, 7497-7504.

(69) Wiberg, K. B.; Hadad, C. M.; LePage, T. J.; Breneman, C. M.; Frisch, M. J. Analysis of the Effect of Electron Correlation on Charge Density Distributions. J. Phys. Chem. 1992, 96, 671-679.

(70) Hanwell, M. D.; Curtis, D. E.; Lonie, D. C.; Vandermeersch, T.; Zurek, E.; Hutchison, G. R. Avogadro: An Advanced Semantic Chemical Editor, Visualization, and Analysis Platform. J. Cheminformatics 2012, 4, 17.

(71) Robertson, A. W.; Allen, C. S.; Wu, Y. A.; He, K.; Olivier, J.; Neethling, J.; Kirkland, A. I.; Warner, J. H. Spatial Control of Defect Creation in Graphene at the Nanoscale. Nat. Commun. 2012, 3, 1144. 
(72) Schultz, T.; Quenneville, J.; Levine, B.; Toniolo, A.; Martínez, T. J.; Lochbrunner, S.; Schmitt, M.; Shaffer, J. P.; Zgierski, M. Z.; Stolow, A. Mechanism and Dynamics of Azobenzene Photoisomerization. J. Am. Chem. Soc. 2003, 125, 80988099.

(73) Yamazaki, K.; Niitsu, N.; Nakamura, K.; Kanno, M.; Kono, H. Electronic Excited State Paths of Stone-Wales Rearrangement in Pyrene: Roles of Conical Intersections. J. Phys. Chem. A 2012, 116, 11441-11450.

(74) Nicholson, C.; Lücke, A.; Schmidt, W.; Puppin, M.; Rettig, L.; Ernstorfer, R.; Wolf, M. Beyond the Molecular Movie: Dynamics of Bands and Bonds During a Photoinduced Phase Transition. Science 2018, 362, 821-825.

(75) Frigge, T.; Hafke, B.; Witte, T.; Krenzer, B.; Streubühr, C.; Samad Syed, A.; MikšićTrontl, V.; Avigo, I.; Zhou, P.; Ligges, M.; von der Linde, D.; Bovensiepen, U.; Horn-von Hoegen, M.; Wippermann, S.; Lücke, A.; Sanna, S.; Gerstmann, U.; Schmidt, W. G. Optically Excited Structural Transition in Atomic Wires on Surfaces at the Quantum Limit. Nature 2017, 544, 207-211.

(76) Jakowski, J.; Morokuma, K. Liouville-von Neumann Molecular Dynamics. J. Chem. Phys. 2009, 130, 224106.

(77) Li, X.; Tully, J. C.; Schlegel, H. B.; Frisch, M. J. Ab initio Ehrenfest dynamics. J. Chem. Phys. 2005, 123, 084106. 